\title{
Influence of Twisting Ratio and Loop Length on Loop Deflection of Flat Fabrics
}

\author{
Jiaxuan Zhang \\ College of Art and Appareluages, Tianjin Polytechnic University \\ Tianjin 300160, China \\ E-mail: dianzizhufu@tom.com \\ Jin Li \\ School of Textiles, Tianjin Polytechnic University \\ Tianjin 300160, China \\ E-mail: jinylee@163.com
}

\begin{abstract}
Twisting ratio and loop length are the main factors to make the loop of plain-knitted fabric deflective. Through taking the wool/acrylic blended knitted fabrics as the experimental material and knitting single plain-knitted fabric on the computer-controlled flat knitting machine, in this article, we validate and compare influences of twisting ratio and loop length on the deflection angle of wale. Through the experiment, conclusions include that the linear relationship is presented between the wale deflection angle and the twist of the bunched line (when the twist of the single yarn is certain), and the loop deflection of the fabric with long length loop will change more obvious with the change of twist, and the rinse can strengthen the tendency of the deflection of wale loop.
\end{abstract}

Keywords: Flat plain-knitted fabric, Loop deflection, Twisting ratio, Loop length

The optimal conformation of fabric is that the vertical loop wale and the horizontal loop course are arranged at a right-angle, but in fact, the deflection tendency always occurs in both for single jersey ( $\mathrm{Wu}, 2004, \mathrm{p} .69-70)$. The deflection and distortion of fabrics puzzle many enterprises all along, because these phenomena not only influence the attractive suit form, but also influence the comfort and increase the difficulty of fabric in its late manufacture.

In early 1980s, through large numbers of experiments, G.W. Smith demonstrated that the loop deflection of fabric was decided by the yarn twist liveliness, the fabric tensity and the number of the knitted machine, and the yarn twist liveliness was the main reason. But the loop deflection direction of fabric is mainly decided by the twist direction of the yarn, and when the $\mathrm{Z}$ yarn is used to knit, the loop of the fabric inclines rightward, and when the $\mathrm{S}$ yarn is used to knit, the loop of the fabric inclines leftward (Chen, 2006, p.13-15).

\section{Experimental material and parameter enactment}

\subsection{Experimental material}

Because the bunched lines with reverse twist has good mightiness, good handle and blare, stable twist circle, small twist shrinking, small single yarn deflection and small elongation, so in this experiment, we adopt 48 pieces of wool/acrylic (30/70) single yarn with $\mathrm{Z}$ twist as the raw material to twist up $48^{2}$ pieces of bunched lines through double reverse. The twist is completed on the thin yarn sample machine made by Tianjin Polytechnic University, and the twist of the single yarn in the experiment is $556.9 \mathrm{tpm}$, and the twist coefficient of the single yarn is 80.38 , and the twist is measured on the Y331 twist testing machine.

\subsection{Confirmation of the twisting ratio}

Various characters of the bunched line are decided by the mutual relationship between the stress distribution and structure of the fibers in the bunched line to large extents. The twist breadth denotes the deflection degree between the fiber and the axes, and approximatively represents the fiber distortion and stress degree, and the blare and the handle of the bunched line are decided by the deflection degree of the fiber on the surface of the bunched line (M.D. de Araujo \& G.W. Smith, 1989, p.350-356).

(1) If the twist breadth of the bunched line equals to the twist breadth of the single yarn, so the upper fiber is parallel with the axes direction, and the twist breadth is zero, and the surface of the bunched line can acquire optimal blare, soft handle and good lengthways wearable capability. Here, the twist coefficient of the bunched line $\alpha_{1}$ and the twist 
coefficient of the single yarn $\alpha_{0}$ have the relationship.

$\alpha_{1}=\frac{\sqrt{2}}{2} \alpha_{0}$

(2) If the twist breadth of the bunched line is double to the twist breadth of the single yarn, so the twist breadths on the inner layer and the outside layer are consistent, and the stress distribution on the inner layer and the outside layer is even, and the mightiness of the bunched line is optimal. Here, $\alpha_{1}=\sqrt{2} \alpha_{0}$.

According to the relationship between the twist breadth of the bunched line and the twist breadth of the single yarn, three twisting ratios in common use are respectively confirmed as $0.414,0.707$ and 1 . From experimental data, we can see that when the twisting ratios are 0.717 and 1 , for the bunched line through adding twist, its self twist direction is the direction $\mathrm{Z}$, and when the twisting ratio is 0.414 , for the bunched line through adding twist, its self twist direction is the direction S. Because we need to find out the critical point that the self twist direction changes in the experiment, so we select a relative smaller twist coefficient 0.2 , here, the self twist direction is the direction $\mathrm{S}$, which can make the data on the both sides are close to balance at the critical point. The parameters of the bunch adding twist are seen in Table 1 .

Because of the uncontrolled factors in the machine and the yarn, differences will occur in the actual twist of the bunched line measured after twist combination and the nominal twist of the bunched line through computation, both represent linear relationship, which is seen in Figure 1 (For convenient for computation, all twists of the bunched line in the following text are nominal twists).

\subsection{Confirmation of knitted parameter}

We take the above bunched line knit to single plain-knitted fabric on the computer-controlled flat knitting machine STOLL CMS 320 TC which machine number is 12 needles/ $25.4 \mathrm{~mm}$. We use the curving yarn depth of the machine in the experiment to control the loop length of the fabric, and according to the branch quantity, twist and flat knitting machine capability, we can choose the curving yarn fabrics with loop depths including $5.6 \mathrm{~mm}, 6.1 \mathrm{~mm}, 6.6 \mathrm{~mm}$ and $7.2 \mathrm{~mm}$

\section{Measurement of the loop deflection angle of the fabric}

Next, we implement the dry looseness disposal and the wet looseness disposal for the fabrics.

\subsection{Measurement of the loop deflection angle under the dry looseness situation}

After 24 hours' balance under standard atmosphere, we measure the course deflection angle of the fabric. Measure 5 data in different places on every fabric and take the average value. The course deflection degree is measured by the course deflection angle $\theta$, which is seen in Figure 2, and $\theta=\arctan (\mathrm{a} / \mathrm{b})$. The deflection angles of various fabrics after balance are seen in Table 2 (Chen, 2006, p.13-15).

\subsection{Measurement of the loop deflection angle under the wet looseness situation}

To every fabric, we implement five-time disposal of "rinse- drying in the shade" and respectively record the loop course deflection angle at a time.

\section{Analysis of experimental results}

Through experimental data, we can see that the loop deflection angle is relative to the twist of the bunched line. The values of the loop deflection angle reduce with the increase of the twist of the bunched line. When the loop deflection direction is right, the angle of the loop deflection reduces with the increase of the twist, and when the loop deflection gradually reduces to $0^{\circ}$, the direction of the loop deflection begin to turn left and the twist continually increases and the angle of the loop deflection also increases.

The loop deflection angle and the twist of the bunched line basically present linear relationship. To the fabric with loop length of $6.6 \mathrm{~mm}$, the linear expression between loop deflection angle and the twist of the bunched line is $\mathrm{y}=-0.1105 \mathrm{x}+28.35$, and the correlation degree $\mathrm{R}^{2}$ is 0.9929 , so according to that, we can predict that when the twist of the bunched line is $256.6 \mathrm{tpm}$, the loop deflection is $0^{\circ}$.

The loop deflection angle is relative to the loop length, and when the twist of the bunched line is certain, the deflection degree of the fabric with long loop length is bigger than the fabric with short loop length, i.e. the loop deflection of the fabric with long loop length has a more obvious change with the change of twist. The relationships between the twist of the bunched line and the loop deflection angle are seen in Figure 3 and Figure 4.

The rinse has certain influences to the loop deflection angle and it can strengthen the deflection tendency that the loop turns right. With the increase of the rinse time (when the rinse exceeds four time), the further deflection tendency of the loop begins to gradually reduce. The relationship between the twist of the bunched line and the loop deflection angle of the fabric is seen in Figure 5. 


\section{Experimental conclusions}

Through the experiment, we can see that the course deflection of the fabric is induced by the inner stress of the yarn adding twist, and the deflection angle and the twist of the bunched line present linear relationship (when the twist of the single yarn is certain), and the loop deflection of the fabric with long loop length changes more obvious with the change of the twist, and the rinse can strengthen the course loop deflection tendency of the fabric.

Comparing the relationship between the loop deflection angle and the twist before the rinse with that after rinse, we find that the twist of the bunched line when the loop deflection is $0^{\circ}$ after rinse is bigger than that before rinse. Therefore, aiming at different rinse frequencies for different fabric in daily life, we can reasonably choose a relationship between rinse times and loop deflection angle and exactly and effectively confirm an optimal twist of the bunched line to make the loop deflection of the fabric achieve minimum.

\section{References}

Chenyan \& Shenwei. (2006). Study of the Wale Deflection of Single Weft Plain Fabric. Knitting Industries. No.1. p.13-15.

Di, Jianfeng. (1997). Influence of Yarn Twisting Back Moment on Loop Deflection of Fabrics. Journal of Textile Research. No.8.

M.D. de Araujo \& G.W. Smith. (1989). Spirality of Knitted Fabrics, Part I: The Nature of Spirality. Textile Research Journal. Vol.59. p.247-256.

M.D. de Araujo \& G.W. Smith. (1989). Spirality of Knitted Fabrics, Part II: The Effect of Yarn Spinning Technology. Textile Research Journal. Vol.59. p.350-356.

Ozkan Celik, Nuray Ucar \& Seniz Ertugrul. (2005). Determination of Spirality in Knitted Fabrics by Image Analyses. Fibres \& Textiles in Eastern Europe. Vol.13. No.3(51).

Peng, Liyun, Cheng, Yukun \& Guo, Xiuping. (2005). Control of Distorted Deformation of Plain Knitted Cotton Garments. Knitting Industries. No.4(4). p.20-22.

P.K. Banerjee \& T.S. Alaiban. (1988). Geometry and Dimensional Properties of Plain Loops Made of Rotor Spun Cotton Yarns, Part II: Spirality of the Wale. Textile Research Journal. Vol.58. p.287-290.

$\mathrm{Wu}$, Honglie. (2004). Methods for Mending Lengthwise Distortion of Single Jersey. Wool textile Journal. No.2. p.69-70.

Yang, Suoting. (2004). Spinning Technology. Beijing: China Textile \& Apparel Press. May, 2004.

Yu, Xufen. (2004). Experimental Technology of Spinning Material. Beijing: China Textile \& Apparel Press. March, 2004.

Zhang, Yiping. (2005). An Analysis of the Skewing and Twisting of Knitted Face Fabric. Shanghai Textile Science \& Technology. No.10. 33(10).

Table 1. Parameter table of the bunch adding twist

\begin{tabular}{|c|c|c|c|c|c|}
\hline $\begin{array}{c}\text { Twist } \\
\text { parameter of } \\
\text { the single } \\
\text { yarn }\end{array}$ & $\begin{array}{c}\text { Twisting } \\
\text { ratio }\end{array}$ & $\begin{array}{c}\text { Twist parameter } \\
\text { of the bunched } \\
\text { line }\end{array}$ & $\begin{array}{c}\text { Nominal twist } \\
\text { of the bunched } \\
\text { line }\end{array}$ & $\begin{array}{c}\text { Actual twist of } \\
\text { the bunched } \\
\text { line }\end{array}$ & $\begin{array}{c}\text { Self twisting } \\
\text { direction of the } \\
\text { bunched line }\end{array}$ \\
\hline 80.38 & 0.2 & 16.08 & 79 & 74.8 & $\mathrm{~S}$ \\
\hline 80.38 & 0.414 & 33.28 & 163 & 14.9 & $\mathrm{~S}$ \\
\hline 80.38 & 0.707 & 56.83 & 278 & 258.8 & $\mathrm{Z}$ \\
\hline 80.38 & 1 & 80.38 & 394 & 359.6 & $\mathrm{Z}$ \\
\hline
\end{tabular}


Table 2. Table of fabric deflection angles (Right deflection is plus, left deflection is negative, and the unit is ${ }^{\circ}$.)

\begin{tabular}{|c|c|c|c|c|}
\hline $\begin{array}{l}\text { Lwist of the length (mm) } \\
\text { bunched line (tpm) }\end{array}$ & 5.6 & 6.1 & 6.6 & 7.2 \\
\hline 79 & 14.6 & 17.0 & 18.4 & 23.5 \\
\hline 163 & 9.5 & 10.5 & 12.1 & 13.7 \\
\hline 278 & -1.0 & -1.9 & -2.6 & -3.3 \\
\hline 394 & -10.9 & -11.8 & -15.5 & -19.8 \\
\hline
\end{tabular}

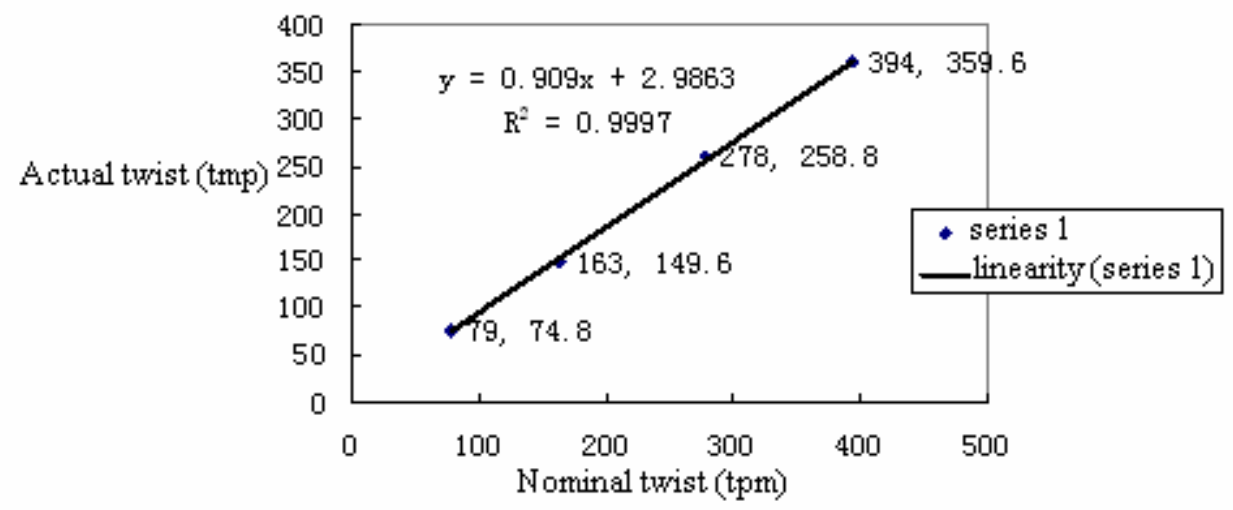

Figure 1. Relationship between the Nominal Twist and the Actual Twist of the Bunched Line

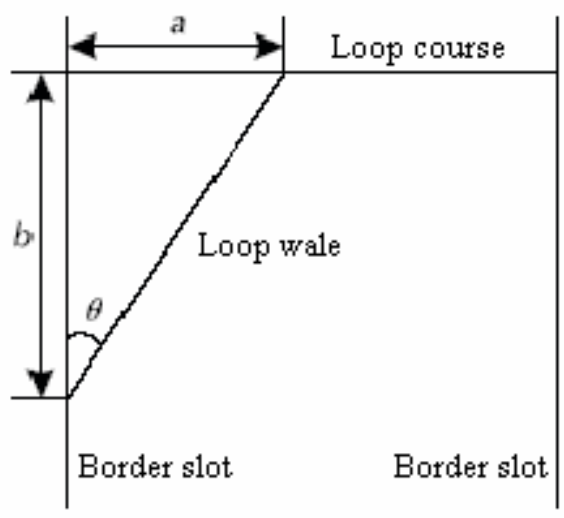

Figure 2. Z Twisting Yarn Wale Deflection 


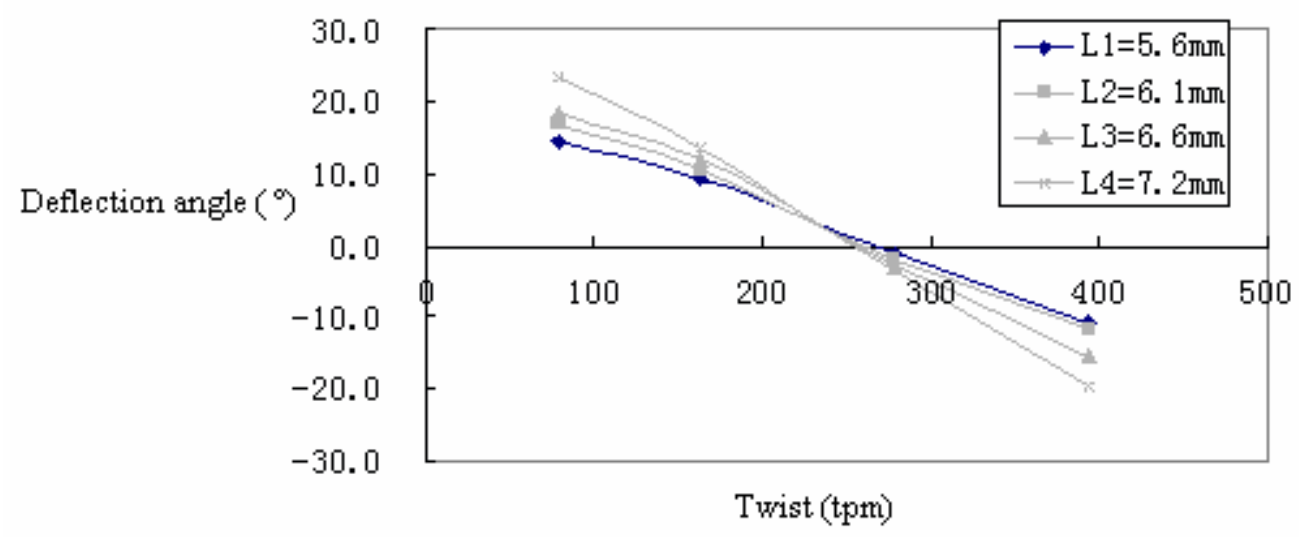

Figure 3. Relationship between Loop Deflection Angle and Twist

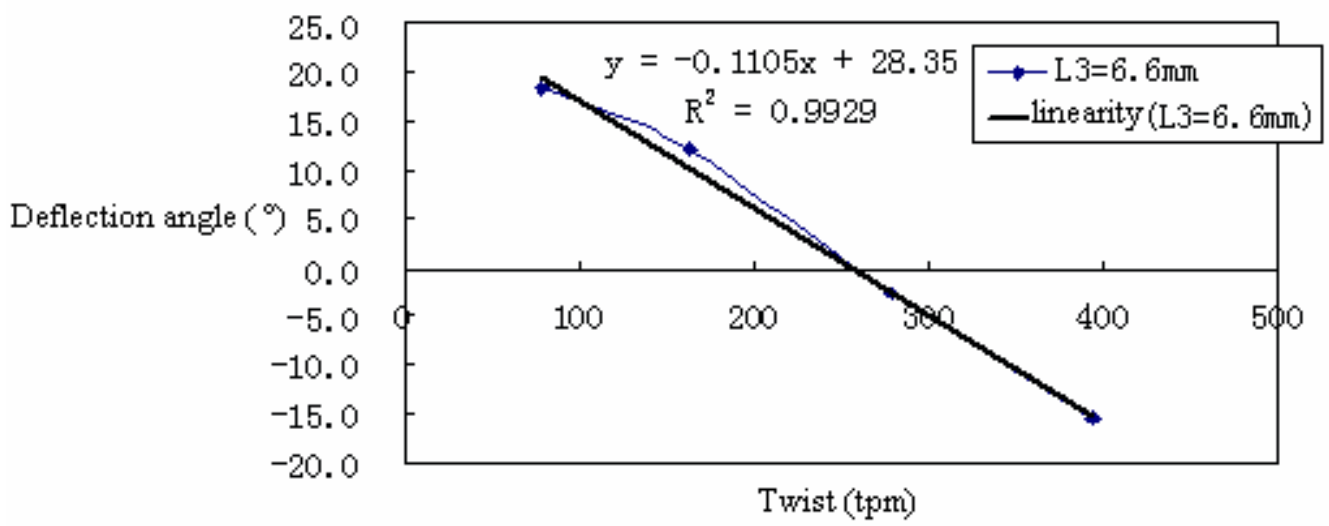

Figure 4. Relationship between Loop Deflection Angle and Twist When the Loop Length is $6.6 \mathrm{~mm}$

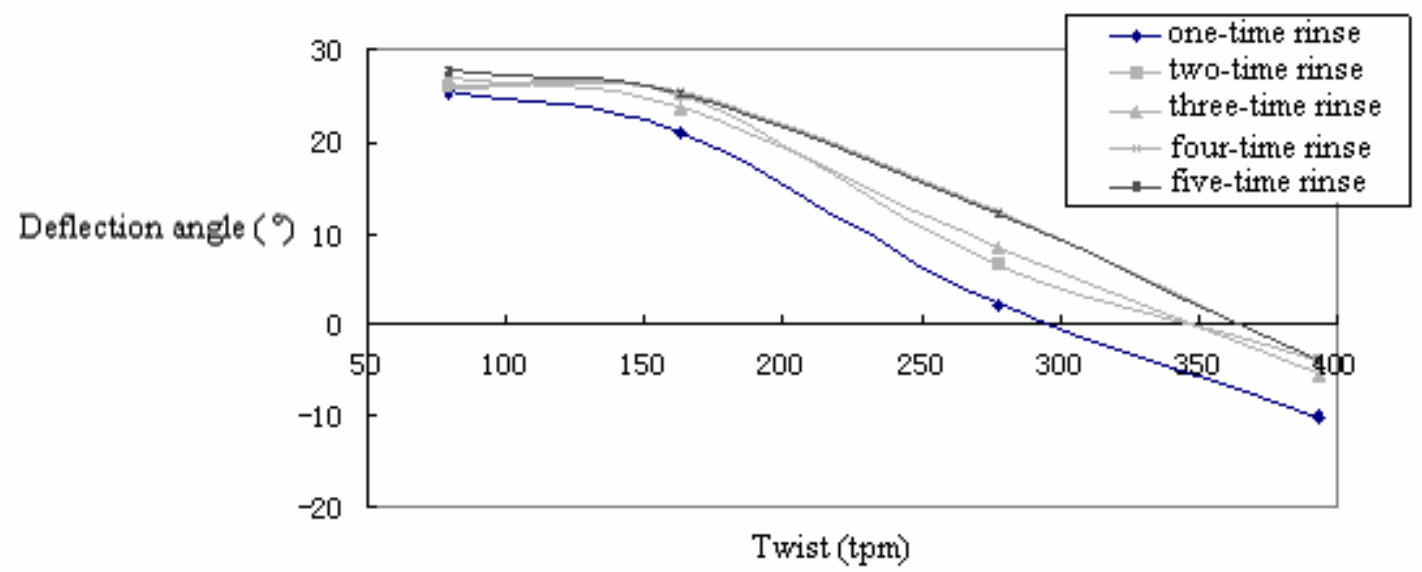

Figure 5. Relationship between Loop Deflection Angle and Twist after Rinse 